\title{
Development of In-Plane Orientation in Pyromellitic Dianhydride-Oxydianiline Polyimide Film on Substrate during Thermal Imidization
}

\author{
Hee-Tak KIm, Sang-Kyung KIm, and Jung-Ki PARK ${ }^{\dagger}$ \\ Department of Chemical Engineering, Korea Advanced Institute of Science and Technology, \\ 373-1 Kusung-dong, Yusung-gu 305-701, Korea \\ (Received July 31, 1998)
}

\begin{abstract}
The development of in-plane orientation in pyromellitic dianhydride-oxydianiline (PMDA-ODA) polyimide film coated on silicon substrate during thermal imidization was investigated by means of FT-IR spectroscopy. The increase in thermal imidization temperature caused the decrease in the degree of in-plane orientation in spite of the increased chain rigidity. It suggests that the chain immobilization limit the development of the in-plane orientation during thermal imidization. The residual solvent was found to decrease the degree of in-plane orientation probably due to the increase of the chain immobilization rate as indicated by the quantitative measurements of the degree of imidization and the residual solvent content as a function of imidization time at various temperatures.
\end{abstract}

KEY WORDS Pyromellitic Dianhydride-Oxydianiline / Thermal Imidization / Chain Rearrangement / In-Plane Orientation / Residual Solvent / Chain Immobilization /

The chain orientation in polyimide coated on substrate is characterized by the term 'in-plane orientation' which means that the director of polyimide chain is parallel to substrate. $^{1-3}$ Since various anisotropic properties of dielectric constant, refractive index, and coefficient of thermal expansion depend on degree of in-plane orientation, ${ }^{4-8}$ the development of in-plane orientation during thermal imidization is a matter of concern.

Since the in-plane orientation of polymer film coated on substrate is originated from the restricted chain rotation near the substrate, a polymer of higher chain rigidity shows stronger tendency for developing in-plane orientation, when polymers of different rigidity are compared. ${ }^{9,10}$ However, we previously found in a study on the effect of imidization condition on the in-plane orientation of polyimide that degree of in-plane orientation of polyimide was, in some cases, found to be higher at lower chain rigidity. ${ }^{11}$ This behavior was explained in terms of the chain immobilization which is caused by the increase of the chain rigidity during thermal imidization. Even though the driving force for developing in-plane orientation would increase as imidization proceeds, the long-range motion of the chain leading to in-plane orientation could be limited by the decreased chain mobility. In this work, we changed the chain immobilization rate for pyromellitic dianhydride-oxydianiline (PMDA-ODA) polyamic acid cast on silicon wafer by varying the imidization temperature and the residual solvent content, and investigated the corresponding changes in the in-plane orientation of polyimide by means of FT-IR spectroscopy to obtain better understanding on the development of in-plane orientation during thermal imidization.

\section{EXPERIMENTAL}

\section{Materials and PAA Cast Preparation \\ Pyralin $^{\circledR}$ (DuPont), the mixture of the polyamic acid (4,4'-oxydiphenylene pyromellitamic acid), $N$-methyl}

pyrrolidinone (NMP), and petroleum ether, was poured into excess methanol to precipitate the polyamic acid. The precipitates were washed with methanol several times and dried at $60^{\circ} \mathrm{C}$ under vacuum for $12 \mathrm{~h}$, and then used to prepare NMP solution of the polyamic acid. NMP was purchased from Aldrich and used as received. NMP solutions of the polyamic acid of various concentrations $(10,15,20 \mathrm{wt} \%)$ were spin-coated on the silicon wafer at $1000 \mathrm{rpm}$. After the spinning, the casts were prebaked at $60^{\circ} \mathrm{C}$ for $3 \mathrm{~h}$.

\section{FT-IR Measurements}

The time-dependent changes of FT-IR spectra of the polyamic acid cast on silicon wafer under isothermal heating were obtained by using the temperaturecontrolled FT-IR kit composed of Bomen 102 spectrometer and heating cell at a resolution of $4 \mathrm{~cm}^{-1}$. The temperature of the kit was controlled to be the imidization temperature before the sample was inserted into the kit. Twenty scans were signal averaged and stored to magnetic disc for further analysis.

To determine the three dimensional chain orientation of the polyimide chain, the polyimide films were carefully peeled from silicon wafer without causing any extension, and the trichroic ratios were calculated from the transmission FT-IR spectra of the films at normal incidence and oblique incidence with polarized IR. The angle for oblique incidence was $30^{\circ}$. To remove the fringe pattern in the spectra which is arisen from the interference among the reflected beams, small amount of mineral oil was coated on the film surface.

\footnotetext{
${ }^{1} H$ NMR Measurement

The ${ }^{1} \mathrm{H}$ NMR spectra for the polyamic acid casts were recorded on Bruker AMX 500 (field strength: $500 \mathrm{MHz}$ ). The solvent used for the sample preparation was dimethyl sulfoxide (DMSO)- $d_{6}$.
}

$\dagger$ To whom all correspondence should be addressed. 


\section{RESULTS AND DISCUSSION}

\section{Effect of Imidization Temperature on In-Plane Orientation}

Figure 1 typically shows the changes in FT-IR spectra of the polyamic acid during thermal imidization. With increasing imidization time, the intensities of the peaks at $1780,1720,1375$, and $725 \mathrm{~cm}^{-1}$ increase. These peaks correspond to the symmetric $\mathrm{C}=\mathrm{O}$ stretching, asymmetric $\mathrm{C}=\mathrm{O}$ stretching, $\mathrm{C}-\mathrm{N}$ stretching, and out-ofplane deformation of the imide ring, respectively. ${ }^{12-14}$ The peaks at 1680 and $985 \mathrm{~cm}^{-1}$ of which intensities decreased with imidization time are originated from the residual NMP molecules. ${ }^{15}$ The itensity of the peak at $1500 \mathrm{~cm}^{-1}$ which corresponds to the $\mathrm{C}-\mathrm{C}$ stretching of $p$-substituted benzene ring does not change with imidization reaction. For that reason, it is generally used as an internal standard by which the effect of film thickness on intensity is excluded. ${ }^{16}$ Among the various peaks from the imide group, the $\mathrm{C}-\mathrm{N}$ stretching vibration at $1375 \mathrm{~cm}^{-1}$ is believed to be the best for the determination of degree of imidization, because the transition moment vector of the $\mathrm{C}-\mathrm{N}$ stretching vibration is parallel to that of the internal standard so that the relative intensity of the peaks is not affected by inplane orientation of the chain. ${ }^{16}$ The degree of imidization was calculated by the following equation

$$
\begin{aligned}
\text { Degree of imidization }(\%) \\
=\left(A_{1375} / A_{1500}\right) /\left(A_{1375} / A_{1500}\right)_{\text {completely imidized one }} \\
\quad \times 100
\end{aligned}
$$

The completely imidized polyimide was obtained by the curing of the polyamic acid cast which contains $20 \mathrm{wt} \%$ of NMP at $350^{\circ} \mathrm{C}$ for $1 \mathrm{~h}$. In order to determine the areas of the peaks at $1500 \mathrm{~cm}^{-1}$ and $1375 \mathrm{~cm}^{-1}$, the spectra in the range of $1200-1560 \mathrm{~cm}^{-1}$ were resolved into several peaks by curve-fitting typically shown in Figure 2. The number of the peak used for the fitting was determined from the second derivative of the raw spectra. Lorenzian peak shape results in best fit for most of the peaks in the spectra except for the broad peak at $1540 \mathrm{~cm}^{-1}$ that was fitted with gaussian peak. The $1500 \mathrm{~cm}^{-1}$ was resolved into two lorentzian peaks and the peak at $1375 \mathrm{~cm}^{-1}$ was fitted with one lorentizian peak. The regression coefficient for each fit always exceeded 0.998 .

Figure 3 shows the changes of degree of imidization with imidization time for the polyimide film isothermally cured at five different temperatures. The imidization reaction rapidly proceeded at the initial stage, and gradually slowed down approaching the eventual level of degree of imidization. This behavior is well explained by the decrease of the chain mobility with imidization reaction. When the glass trasition temperature $\left(T_{\mathrm{g}}\right)$ of the reacting medium reaches imidization temperature, the chain becomes immobile and the amic acid unit would be difficult to attain favorable conformation for imidization, which results in the lowering of the reaction rate. ${ }^{17}$ Unfortunately, the experimental confirmation of the above consideration by measuring the $T_{\mathrm{g}}$ with thermal analysis is impossible, because the unreacted amic acid is transformed into imide near the $T_{\mathrm{g}}$, resulting in the gradual increase of $T_{\mathrm{g}}$ during the measurement. The
C-C stretching of benzene ring

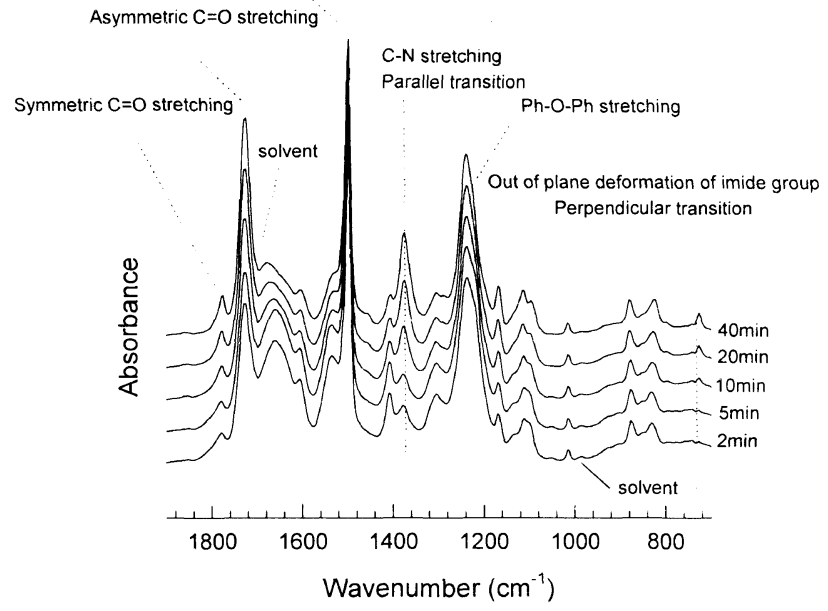

Figure 1. Time dependent changes of FT-IR spectra for PMDA-ODA polyamic acid during thermal imidization at $150^{\circ} \mathrm{C}$.

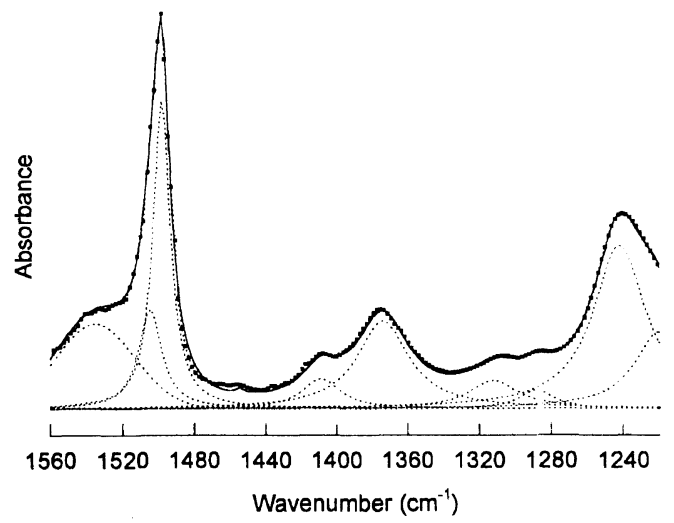

Figure 2. Typical curve fitting results of the FT-IR spectrum of the polyimide in the region of $1200-1560 \mathrm{~cm}^{-1}$ (imidization condition: $180^{\circ} \mathrm{C}$ for $12 \mathrm{~min}$ ).

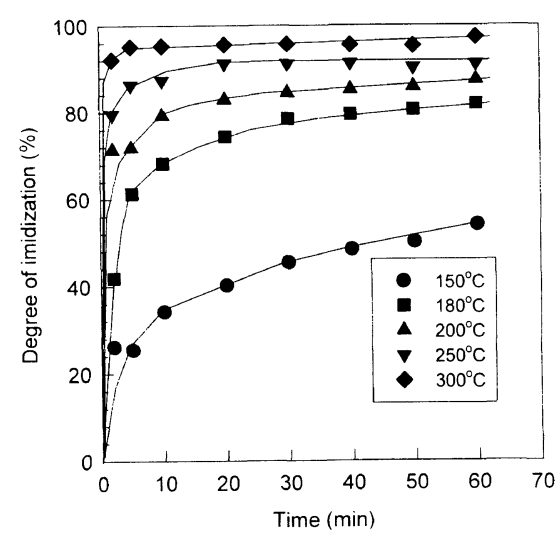

Figure 3. Change of degree of imidization with imidization time at various imidization temperatures (casting solution: $20 \mathrm{wt} \%$ solution of PMDA-ODA polyamic acid).

initial imidization rate and the final level of degree of imidization were found to increase with imidization temperature. At the higher imidization temperature, the imidization rate started to slow down at the earlier stage, indicating the faster chain immobilization.

The chain orientation of the polyimide segment was quantitatively measured by polarized FT-IR spectro- 


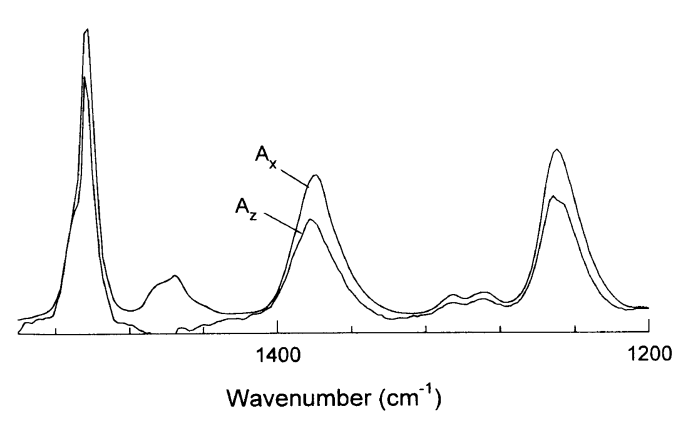

Figure 4. Comparison of $A_{x}$ and $A_{z}$ FT-IR spectra for polyimide film.

Table I. $\quad R_{z y}$ and $\left\langle\cos ^{2} \theta\right\rangle$ for the C-N stretching vibration $\left(1375 \mathrm{~cm}^{-1}\right)$ of the polyimide imidized at various temperatures

\begin{tabular}{ccc}
\hline Temp & $R_{z y}$ & $\left\langle\cos ^{2} \theta\right\rangle$ \\
\hline $180^{\circ} \mathrm{C}$ & 0.180 & 0.847 \\
$200^{\circ} \mathrm{C}$ & 0.187 & 0.842 \\
$250^{\circ} \mathrm{C}$ & 0.194 & 0.838 \\
$300^{\circ} \mathrm{C}$ & 0.287 & 0.777 \\
$350^{\circ} \mathrm{C}$ & 0.541 & 0.649 \\
\hline
\end{tabular}

scopic technique. The orientation in three dimensions is completely characterized if the $A_{z}$ component of the absorption is determined together with the $A_{x}$ and $A_{v}$ components, where $x$ and $y$ represent two directions both parallel to the substrate and perpendicular with each other and $z$ indicates the normal direction to the substrate (thickness direction). $A_{x}$ and $A_{y}$ can be easily obtained by using polarized FT-IR spectroscopy at normal incidence. ${ }^{18-21}$ The thickness direction absorbance is known to be obtained by tilting the sample at a certain angle $\alpha$, and determining the component in the $z$ direction through the relationship. ${ }^{18-21}$

$$
A_{z}=\frac{A_{\alpha} \sqrt{1-\sin ^{2} \alpha / n^{2}}-A_{y}}{1-\sin ^{2} \alpha / n^{2}}+A_{y}
$$

where $\alpha$ is the tilting angle of the sample for oblique incidence (the angle between the direction of incident IR beam and the direction normal to the substrate) and $n$ is the refractive index of the film. In our experiments, $\alpha$ was $30^{\circ}$ and $n$ of the film along the incidence direction was 1.7 which is determined from the refractive index ellipsoid with the reported $n_{\mathrm{e}}$ and $n_{\mathrm{o}}$ values. $^{2}$

We investigated the orientation of the transition moment of $\mathrm{C}-\mathrm{N}$ stretching appeared at $1375 \mathrm{~cm}^{-1}$. Since the direction of the transition moment for the $\mathrm{C}-\mathrm{N}$ stretching vibration is parallel to the director of imide segment, it can represent the orientation of the imide segment. The $A_{x}$ and $A_{y}$ spectra were found to be exactly the same with each other, which indicates that there was no preferential orientation over the substrate plane. However, the $A_{z}$ and $A_{x}$ were quite different as shown in Figure 4. It indicates the existence of an anisotropy in the thickness direction. Table I lists the value for $R_{z y}$, the ratio of $A_{z}$ and $A_{y}$, which informs the average orientation of the transition moment of the $\mathrm{C}-\mathrm{N}$ stretching. The angle of the transition moment from the substrate $(\theta)$ is related with $R_{z y}$ as given in eq 3 .

$$
R_{z y}=\frac{A_{z}}{A_{y}}=\left\langle\tan ^{2} \theta\right\rangle=\frac{1-\left\langle\cos ^{2} \theta\right\rangle}{\left\langle\cos ^{2} \theta\right\rangle}
$$

The values of $R_{z y}$ determined from the peak at $1375 \mathrm{~cm}^{-1}$ were found to be lower than unity irrespectively of imidization temperature, which indicates the developed in-plane orientation of the polyimide chain. The value of $R_{z y}$ and the $\left\langle\cos ^{2} \theta\right\rangle$ were found to increase and decrease with imidization temperature respectively, representing the lower degree of in-plane orientation for higher imidization temperature.

According to Figure 3, the chain rigidity is higher for the film imidized at higher temperature, and thus the driving force for developing in-plane orientation increases with imidization temperature, which would result in the increase of in-plane orientation. However, the observed results were contrary to this consideration. Rather, the degree of in-plane orientation seems to be mainly dependent on chain immobilization rate. The chain rearrangement to cause in-plane orientation would require a certain degree of chain mobility, and thus when the chain becomes immobilized by the increase of $T_{\mathrm{g}}$, the development of in-plane orientation would be limited. Because the chain immobilization is faster at higher temperature, the lower in-plane orientation can be resulted in at the higher imidization temperature.

It is important to note that even though the chain immobilization rate is found to mainly affect the development of in-plane orientation during thermal imidization, the rigidity of the chain is still a requisite for in-plane orientation. Because the degree of imidization reached $80 \%$ even at $180^{\circ} \mathrm{C}$ for PMDA-ODA, the chains would be stiff enough to cause in-plane orientation. In the case of poly(vinyl chloride), which is one of the typical flexible polymer, quite lower degree of in-plane orientation was observed compared to polyamic acids and polyimides, ${ }^{10}$ demonstrating the role of chain rigidity on in-plane orientation. In our previous work on polyimide derived from cyclobutanedianhydride and 2,2-bis(4-aminophenoxyphenyl)propane (CBDABAPP) polyimide, ${ }^{11}$ in-plane orientation was lower at $150^{\circ} \mathrm{C}$ than at 200 and $250^{\circ} \mathrm{C}$. In that case, the imidization level at $150^{\circ} \mathrm{C}$ was only $15 \%$, which is too small to be effective for in-plane orientation and thus lower in-plane orientation at lower imidization temperature could be resulted in.

In order to support the chain immobilization at the early stage of thermal imidization, which was deduced from the stop of imidization reaction, the changes of the chain orientation with imidization time were qualitatively traced. Since FT-IR spectra are so quickly changed with time, the trichroic technique which requires both normal and oblique incidence cannot be applied. Instead, we investigated time dependent changes of the absorbance ratio of the two peaks centered at $725 \mathrm{~cm}^{-1}$ and $1375 \mathrm{~cm}^{-1}$, which can be qualitative measurement for molecular orientation.

The transition moment of the peaks at $1375 \mathrm{~cm}^{-1}$ is parallel to the chain direction, whereas that of the peak at $725 \mathrm{~cm}^{-1}$ is perpendicular to the chain direction. The absorbance of IR band is proportional to the inner product of the transition moment vector of the dipole and the electric vector of the incident IR beam. ${ }^{22}$ As 


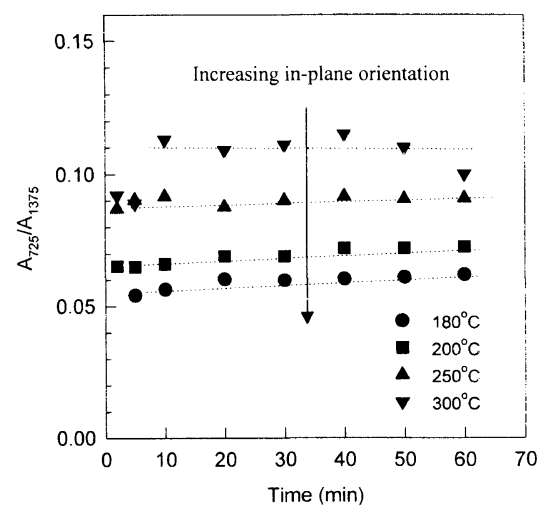

Figure 5. Change of $A_{725} / A_{1375}$ with imidization time at various imidization temperatures (casting solution: $20 \mathrm{wt} \%$ ).

the in-plane orientation increases, the angle between the transition moment vector and the electric vector of the incident beam decreases for parallel transition and increases for perpendicular transition. The areas of these two peaks also vary with the concentration of the imide group, i.e., the degree of imidization as well as in-plane orientation. However the value of $A_{725} / A_{1375}$, the area ratio of the peaks centered at 725 and $1375 \mathrm{~cm}^{-1}$, is dependent only on in-plane orientation not on degree of imidization. Since the two peaks at 725 and $1375 \mathrm{~cm}^{-1}$ are originated from the same imide group, the contributions from degree of imidization to the areas of these two peaks are ruled out in this ratio. Thus, the value of $A_{725} / A_{1375}$ can be indicative of the in-plane orientation and should decrease with increasing the inplane orientation.

The plots of $A_{725} / A_{1375}$ as a function of imidization time for five different imidization temperature are given in Figure 5. The data were obtained from the in situ FT-IR spectroscopy during thermal imidization of the polyamic acid casts prepared from $20 \mathrm{wt} \%$ solution. The absorbance ratio was not determined below $180^{\circ} \mathrm{C}$, because the area of the peak at $725 \mathrm{~cm}^{-1}$ was too small in the early stage of imidization below $180^{\circ} \mathrm{C}$ as can be seen Figure 1. The value for $A_{725} / A_{1375}$ is increased with increasing imidization temperature which means the decrease of the degree of in-plane orientation with imidization temperature. These results are consistent with those obtained from trichroic method. The ratios were found to be nearly unchanged with imidization time from the very early stage of the thermal imidization. It indicates that the chain rearrangement stops at the beginning stage of the thermal imidization as a result of chain immobilization.

\section{Effect of Residual Solvent Content on In-Plane Orientation}

As shown above, the increase of imidization temperature enhances chain immobilization rate. Since the residual solvent can increase thermal imidization rate, the increase of the chain immobilization rate with the increase of the residual solvent content and the consequent decrease of the in-plane orientation are expectable. In order to investigate the effect of residual solvent, the three polyamic acid casts which are different in residual solvent content were prepared, and the degree of imidization and in-plane orientation were traced during thermal imidization.

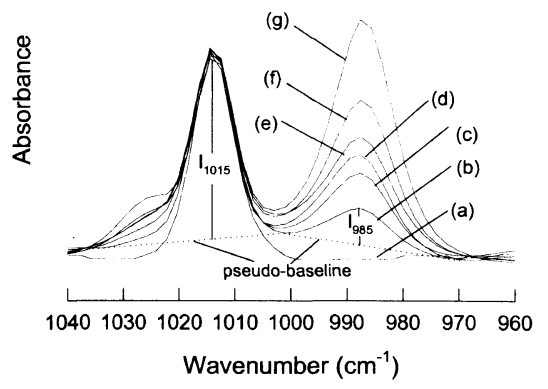

Figure 6. FT-IR spectra in the region of $960-1040 \mathrm{~cm}^{-1}$ of the solvent-containing polyamic acids with various solvent contents $(\sharp N M P /$ amic acid unit $)=$ a) 0 , b) 1.0, c) 1.3, d) 1.5, e) 1.7, f) 2.2, g) 3.2 .

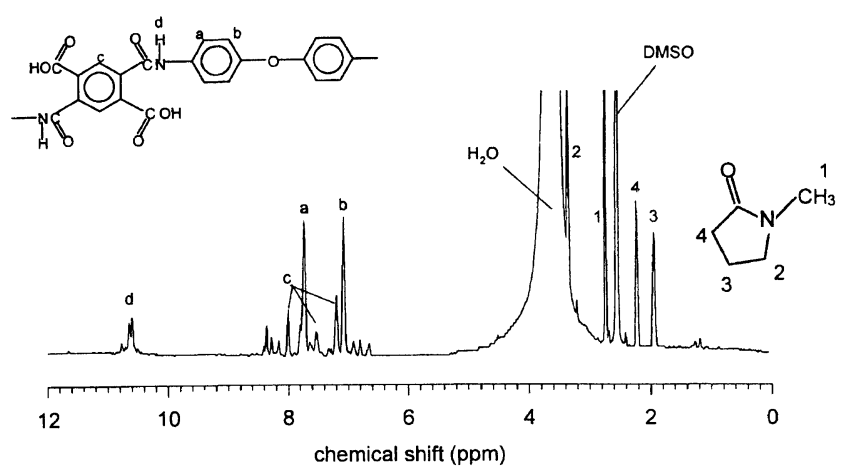

Figure 7. ${ }^{1} \mathrm{H}$ NMR spectra of the PMDA-ODA polyamic acid cast containing the residual NMP.

The exact determination of the amount of residual solvent in the film from FT-IR spectra requires the calibration equation which relates the residual solvent content and the relative peak area from NMP found in FT-IR spectra of the casts. The quantification of the residual solvent content directly from FT-IR spectra for the casts provides in situ monitoring of the residual solvent content during thermal imidization. Figure 6 compares the FT-IR spectra in the range of 960-1040 $\mathrm{cm}^{-1}$ for the polyamic acids of various solvent contents. The peak at $1015 \mathrm{~cm}^{-1}$ corresponds to the skeleton vibration of benzene, and the peak at $985 \mathrm{~cm}^{-1}$ comes from the residual NMP. ${ }^{15}$ The height ratio of the two peaks $\left(I_{985} / I_{1015}\right)$ was used for the relative determination of the residual solvent content. The height of the peak was determined with a pseudo-baseline as shown in Figure 6. Exact amount of residual solvent content in the cast was determined from ${ }^{1} \mathrm{H}$ NMR spectra of the casts. Figure 7 shows the ${ }^{1} \mathrm{H}$ NMR spectra of PMDAODA polyamic acid cast containing NMP. The detailed peak assignments are given in this figure. The complexity of the peaks from the proton in PMDA part (marked as 'c') is due to the presence of the cis and the trans isomers of the amic acid unit. From the relative areas of the peaks from the methylene proton of NMP and the amide proton of the polyamic acid, the number of the NMP molecules per monomeric unit of polyamic acid was determined. From these data, the relationship between the height ratio $\left(I_{985} / I_{1015}\right)$ and residual solvent content was made as given in eq 4 .

$$
y=5.54 x-5.26 x^{2}+1.76 x^{3}
$$

where $y$ and $x$ correspond to the number of NMP molecules per monomeric unit in the film and the height 
Table II. Residual solvent content of the polyamic acid casts prepared from the NMP solutions of different concentration

\begin{tabular}{cc}
$\begin{array}{c}\text { Concentration of the } \\
\text { cast solution }\end{array}$ & $\begin{array}{c}\text { Number of NMP molecules } \\
\text { per amic acid unit }\end{array}$ \\
\hline $20 \mathrm{wt} \%$ & 2.2 \\
$15 \mathrm{wt} \%$ & 1.8 \\
$10 \mathrm{wt} \%$ & 1.5 \\
\hline
\end{tabular}

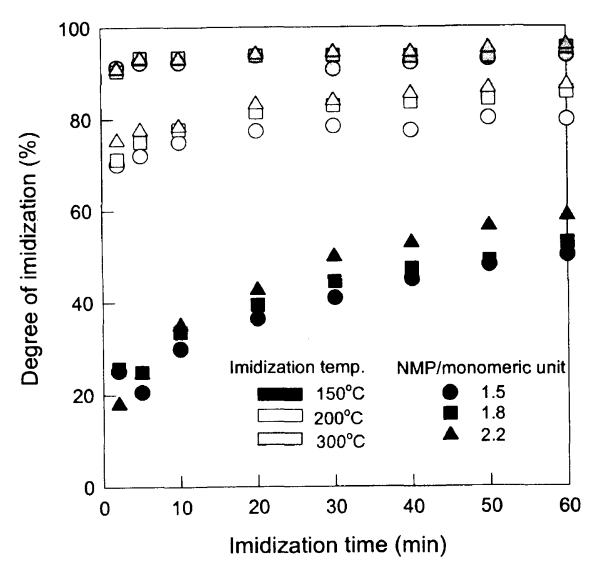

Figure 8. Change of degree of imidization with imidization time at various imidization temperatures for the polyamic acids with different residual solvent content.

ratio determined from the FT-IR spectra respectively. Since a pseudo-baseline was used for the determination of the height of the peak, the height ratio is dependent not only the concentration of the residual solvent but also the height of the neighboring peaks. For that reason, the peak height ratio is not proportional to the solvent content. The initial residual solvent contents calculated from ${ }^{1} \mathrm{H}$ NMR spectra were listed in Table II.

Figure 8 shows the comparison of the degree of imidizations among the three polyamic acid casts with different residual solvent content. As a whole, the higher degree of imidization was obtained for the higher initial residual solvent content, which is well explained by the difference in plasticizing effect of the residual solvent to enhance thermal imidization. ${ }^{23,24}$ The difference of degree of imidization with different solvent content was not apparent at $300^{\circ} \mathrm{C}$. It is probably attributed to the high evaporation rate of the residual solvent at $300^{\circ} \mathrm{C}$, which would diminish the effect of the residual solvent on imidization reaction. The explanations for this behavior are partly supported by comparison of the time-dependent change of the residual solvent content as shown in Figure 9. The change of the solvent content in the casts with imidization time at various imidization temperatures determined by eq 4 is given in this figure. Above $200^{\circ} \mathrm{C}$, most of the solvents are evaporated within few minutes after the start of the heating. The residual solvent content which remained after the thermal imidization, was also dependent on imidization temperature. Nearly no residual solvent is remained in the cast above $200^{\circ} \mathrm{C}$.

Figure 10 represents the plot of $A_{725} / A_{1375}$ as a function of degree of imidization. The open symbol, solid symbol, and half filled symbol represent the data from the polyamic acid casts prepared from 20,15 , and $10 \mathrm{wt} \%$

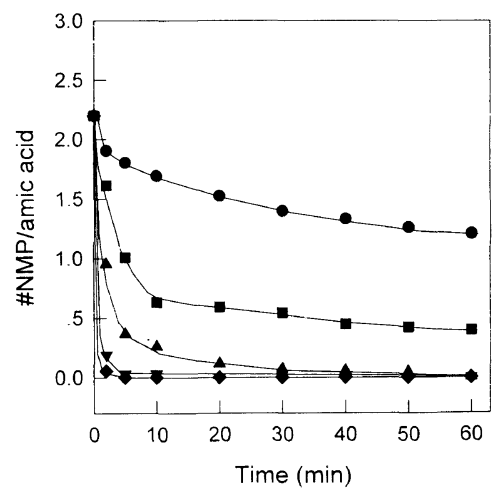

Figure 9. Change of residual solvent content with imidization time at various temperatures., $150^{\circ} \mathrm{C} ; \boldsymbol{\square}, 180^{\circ} \mathrm{C} ; \boldsymbol{\Delta}, 200^{\circ} \mathrm{C} ; \nabla, 250^{\circ} \mathrm{C}$; $\bullet, 300^{\circ} \mathrm{C}$.

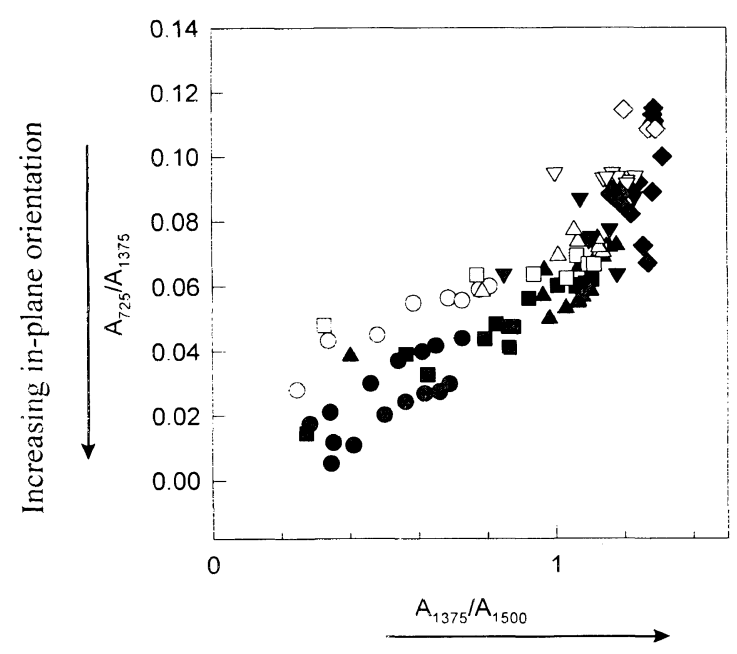

Increasing degree of imidization

Figure 10. Plot of $A_{725} / A_{1375}$ as a function of degree of imidization (open symbol: obtained from the PAA cast prepared from $20 \mathrm{wt} \%$ NMP solution: solid symbol, $15 \mathrm{wt} \%$ solution; half filled symbol, $10 \mathrm{wt} \%$ solution). $\bigcirc, 150^{\circ} \mathrm{C} ; \boldsymbol{\square}, 180^{\circ} \mathrm{C} ; \boldsymbol{\Delta}, 200^{\circ} \mathrm{C} ; \nabla, 250^{\circ} \mathrm{C} ; \diamond$, $300^{\circ} \mathrm{C}$.

NMP solution, respectively. As a result, the in-plane orientation decreases with increasing degree of imidization and the lower degree of in-plane orientation was observed for the higher residual solvent content at a same degree of imidization. The difference in in-plane orientation with residual solvent content can be explained in terms of the difference in chain immobilization rate. Due to the faster imidization by the plasticizing effect of the residual solvent, the chain immobilization would be faster for the higher residual solvent content, resulting in the lower in-plane orientation. It seems that the effect of initial residual solvent content on the in-plane orientation is somewhat diminished at high temperatures above $200^{\circ} \mathrm{C}$. It would be due to the high evaporation rate of the residual solvent at the high imidization temperature as indicated by Figure 9.

On the other hand, it is possible that the difference in initial degree of in-plane orientation of polyamic acid film causes the difference in chain orientation of imide group. We measured the $R_{z y}$ of the peak at $1500 \mathrm{~cm}^{-1}$, of which transition moment is proportional to the chain axis, using trichroic method. The values for $R_{z y}$ was 0.65 , 0.66 , and 0.69 for $\mathrm{NMP} /$ monomeric unit $=1.5,1.8$, and 
2.2 respectively. The in-plane orientation of polyamic acid slightly decreased with the residual solvent content. Even though the difference is small, the possibility that the effect of the initial orientation of polyamic acid on in-plane orientation cannot be excluded. Much work should be needed to elucidate this consideration.

Acknowledgment. This work was supported by the Center for Advanced Functional Polymers.

\section{REFERENCES}

1. T. P. Russel, H. Gugger, and J. D. Swalen, J. Polym. Sci., Polym. Phys. Ed., 21, 1745 (1983).

2. K. Nakahawa, J. Appl. Polym. Sci., 41, 2049 (1990).

3. T. S. Chow, J. Polym. Sci., Polym. Phys. Ed., 25, 137 (1987).

4. J. C. Coburn and M. T. Pottiger, in "Advances in Polyimide Science and Technology," C. Feger, M. M. Khojasteh, and M. S. Htoo, Ed., Technomic, Lancaster, PA, 1993, p 360.

5. T. P. Russel, H. Gugger, and J. D. Swalen, J. Polym. Sci., Polym. Phys., 32, 825 (1994).

6. M. T. Pottiger and J. C. Coburn, Mater. Res. Soc. Symp. Proc., 227, 187 (1991).

7. M. T. Pottiger and J. C. Coburn, Polym. Mater. Sci. Eng., 66 , 194 (1992)

8. L. Lin and S. A. Bidstrup, Polym. Mater. Sci. Eng., 66, 265 (1992).
9. H. Nomura and M. Asano, Jpn. J. Appl. Phys., 32, 3933 (1993).

10. M. Hasegawa, T. Matano, Y. Shindo, and T. Sugimura, Macromolecules, 29, 7987 (1996).

11. H. T. Kim and J. K. Park, Polym. J., 29, 1002 (1997).

12. H. Ishida, S. T. Wellinghoff, E. Baer, and J. L. Koenig, Macromolecules, 13, 826 (1980).

13. E. B. Wilson, Jr., Phys. Rev., 45, 706 (1934).

14. W. H. Tsai, J. T. Young, F. J. Boerio, and P. Phong, Langmuir, 7, 745 (1991).

15. B. Thomson, Y. Park, and P. C. Painter, Macromolecules, 22, 4159 (1989)

16. C. A. Pryde, J. Polym. Sci., Polym. Chem. Ed., 27, 711 (1989).

17. L. A. Laius, M. I. Bessonov, Ye. V. Kallistova, N. A. Adrova, and F. S. Florinskii, Polym. Sci. USSR, A9, 2470 (1967).

18. J. K. Koenig, S. W. Cornell, and D. E. Witenhafen, J. Polym. Sci., A2, 301 (1967)

19. L. J. Fina and J. K. Koenig, J. Polym. Sci., Polym. Phys. Ed., 24, 2509 (1986).

20. L. J. Fina and J. K. Koenig, J. Polym. Sci., Polym. Phys. Ed., 24, 2525 (1986)

21. L. J. Fina and J. K. Koenig, J. Polym. Sci., Polym. Phys. Ed., 24, 2541 (1986)

22. J. K. Koenig, in "Spectroscopy of Polymers," American Chemical Society, 1992, p 26

23. M. J. Brekner and C. Feger, J. Polym. Sci., Polym. Chem. Ed., 25, 2005 (1987)

24. M. J. Brekner and C. Feger, J. Polym. Sci., Polym. Chem. Ed., 25, 2479 (1987). 